\title{
Quantum Evolution and Classical Flow in Complex Phase Space
}

\author{
S. Graffi ${ }^{1}$ and A. Parmeggiani ${ }^{2}$ \\ ${ }^{1}$ Dipartimento di Matematica, Università di Bologna, I-40127 Bologna, Italy \\ ${ }^{2}$ Department of Mathematics, Princeton University, Princeton, NJ 08544, USA
}

\begin{abstract}
For a class of holomorphic perturbations of the harmonic oscillator in $n$ degrees of freedom a local solution of the time-dependent Schrödinger equation in the Bargmann representation is constructed which pointwise propagates, to leading order in $\hbar$, along the classical trajectories in complex phase space.
\end{abstract}

\section{Introduction and Statement of the Result}

The relation between the quantum flow, i.e. the solutions of the time-dependent Schrödinger equation, and the corresponding classical Hamiltonian flow is a very old problem of quantum mechanics. It is well known that the strongest possible relation between classical and quantum flow, (in the sense that the classical evolution determines the quantum one exactly, not just at leading order in $h$, and pointwise, not just in $L^{2}$ sense) takes place for the coherent states of a system of linear oscillators, i.e. for a system of linear oscillators provided their quantum evolution is described in the Bargmann representation of the canonical commutation rules.

Consider indeed, for $(q, p) \in T^{*} \mathbf{R}^{n} \cong \mathbf{R}^{2 n},\left\{p_{i}, q_{j}\right\}=\delta_{i j}$, the classical Hamiltonian of a system of $n$ independent oscillators of unit frequencies:

$$
H_{0}(p, q)=\frac{1}{2} \sum_{k=1}^{n}\left(p_{k}^{2}+q_{k}^{2}\right) .
$$

The transformation:

$$
\begin{gathered}
C: z_{k}=\frac{1}{\sqrt{2}}\left(q_{k}-i p_{k}\right), \bar{z}_{k}=\frac{1}{\sqrt{2}}\left(q_{k}+i p_{k}\right) \\
C^{-1}: q_{k}=\frac{1}{\sqrt{2}}\left(z_{k}+\bar{z}_{k}\right), p_{k}=\frac{1}{\sqrt{2} i}\left(\bar{z}_{k}-z_{k}\right)
\end{gathered}
$$


is a linear complex canonical transformation of $\mathbf{C}^{2 n}$ into itself such that $T^{*} \mathbf{R}^{n}$ is mapped one-to-one onto the real analytic submanifold $\Lambda$ of $\mathbf{C}^{2 n}$ defined as $\Lambda=\left\{(z, \zeta) \in \mathbf{C}^{2 n} ; \zeta=-i \bar{z}\right\}$. The transformed Hamiltonian $K_{0}(z, \bar{z})=H_{0}\left(C^{-1}(z, \bar{z})\right)$ takes the form:

$$
K_{0}(z, \bar{z})=\sum_{k=1}^{n} z_{k} \bar{z}_{k}
$$

and since $\left\{z_{i}, \bar{z}_{j}\right\}=i \delta_{i j}$ the Hamilton equations in the canonical coordinates $(z, \bar{z})$ are:

$$
\frac{d z}{d t}=i \nabla_{\bar{z}} K_{0}=i z ; \quad \frac{d \bar{z}}{d t}=-i \nabla_{z} K_{0}=-i \bar{z}
$$

with the initial conditions $z(0)=w, \bar{z}(0)=\bar{w}$, whence the phase-space flow:

$$
w \mapsto z=w e^{i t}, \bar{w} \mapsto \bar{z}=\bar{w} e^{-i t}
$$

for any initial condition $(w, \bar{w}) \in \mathbf{C}^{2 n}$. Here of course $z e^{i t}=\left(z_{1} e^{i t}, \ldots, z_{n} e^{i t}\right)$.

The generating function of $C$ is

$$
\varphi(z, q)=\frac{1}{2} i(\langle z, z\rangle+\langle q, q\rangle-2 \sqrt{2}\langle z, q\rangle),
$$

where $\langle z, z\rangle=\sum_{k=1}^{n} z_{k} \bar{z}_{k}$.

Its (normalized) exponential in unit $h$ (here $h=\hbar=\frac{h}{2 \pi}$, where $h$ is the Planck constant $), A(z, q)=(\sqrt{\pi} h)^{-n / 2} \exp \left(i h^{-1} \varphi(z, q)\right)$ is the integral kernel defining the unitary map $U$ :

$$
(U \psi)(z)=\int_{\mathbb{R}^{n}} A(z, q) \psi(q) d q, \quad \psi \in L^{2}\left(\mathbb{R}^{n} ; d q\right)
$$

between $L^{2}\left(\mathbb{R}^{n} ; d q\right)$ and the Hilbert space of holomorphic functions introduced by Bargmann [Ba]:

$$
\mathscr{F}_{n}=\left\{u: \mathbf{C}^{n} \rightarrow \mathbf{C} ; u \text { is holomorphic, } \int_{\mathbb{R}^{2 n}}|u(z)|^{2} e^{-h^{-1}|z|^{2}} L(d z)<+\infty\right\},
$$

where $L(d z)$ is the Lebesgue measure in $\mathbf{C}^{n} \cong \mathbf{R}^{2 n}$.

In this representation of the canonical commutation rules the classical canonical variables $z_{k}$ and $\bar{z}_{k}$ are quantized by the maximal multiplication operator by $z_{k}$ and the maximal differentiation operator $h \frac{\partial}{\partial z_{k}}$ in $\mathscr{F}_{n}$, respectively. Therefore the time-dependent Schrödinger equation generated by the classical Hamiltonian $K_{0}$ reads:

$$
i h \partial_{t} f(t, z)=h\left\langle z, \nabla_{z}\right\rangle f(t, z) .
$$

Its solution with initial value $f(t, z)_{\mid t=0}=f_{0}(z)$ is of course, for all times:

$$
f(t, z)=f_{0}\left(z e^{-i t}\right) \text {. }
$$


This formula shows that for the linear oscillators the classical dynamics and the quantization "commute:" namely, the quantum evolution is obtained just by letting the argument of the initial datum evolve along its Hamiltonian flow.

A natural question arising in this context is therefore to what extent this result holds beyond this very particular case in which the classical flow [see (1.5)] is globally periodic with period independent of the initial conditions ("isochrony" property; the discussion holds of course unchanged in the quasi-periodic case, i.e. when the frequencies of the independent oscillators are rationally independent and the resulting flow is quasi-periodic). The purpose of this paper is to show that, just by working out some details in the Sjöstrand construction [Sj] of the Fourier Integral Operator (FIO) with complex phase on complex domains [up to the variant in taking the analytic stationary phase expansion described in Remark (v) after the statement of the Theorem], it is possible to extend (1.9), to leading order in $h$ (but all corrections in ascending power of $h$ computed) and locally (both in phase space and in time) to a class of more realistic potentials diverging at infinity. The anisochrony of the classical motions prevents the flow $w \mapsto z(t, w)$ from being a global diffeomorphism of $\mathbf{C}^{n}$ : this allows us to construct [modulo $O\left(h^{\infty}\right)$ ] only a distribution solution, namely a locally holomorphic solution which does not belong to the Bargmann space, and furthermore only for short times, since the long time behaviour of the motions is governed by the perturbation, unlike the asymptotically free case recalled below. This local solution enjoys however the property of "pointwise propagation along the classical trajectories" which is the natural generalization of the above result to the non-isochronous case: its value $u(t, z)$ at the point $z$ and at time $t$ is obtained, to lowest order in $h$, just by evaluating the initial Cauchy datum at $w(t, z)$, where $w(t, z)$ is the (backward) evolution at time $t$ under the classical Hamiltonian flow of initial datum $(z, \bar{z})$.

Results of this type are long known when the potentials vanish at infinity, i.e. in the asymptotically free case ([Ya1, Ya 2]; see also [RoTa]). To leading order in $h$ the quantum evolution (in $L^{2}$ sense) at large times can be obtained, up to a well determined phase factor, essentially by letting the argument of the (microlocalized) initial datum at $t=-\infty$ evolve along its corresponding classical flow. The considerably more difficult case in which the potentials diverge (positively) at infinity has been considered only more recently within the techniques of microlocal analysis, notably, in addition to [Sj], by Chazarain [Ch], HelfferRobert [HeRo1, HeRo2], Robert-Petkov (RoPe], Tamura [Ta], Zelditch [Ze]. These last papers however deal mostly with the problem of relating the qualitative properties of classical motions, such as periodicity, to the spectrum of the corresponding Schrödinger operator, except that of Zelditch who obtained for a class of bounded perturbations of the harmonic oscillators a result of reconstruction of singularities along the corresponding unperturbed classical Hamiltonian flow, in analogy with the propagation of singularities along the bicharacteristics for hyperbolic PDE.

Let us turn to state the present result. Consider the set of classical Hamiltonians of the form:

$$
H(p, q, \varepsilon)=H_{0}(p, q)+\varepsilon V(q),
$$


where the assumptions on the potential $V(q)$ are as follows:

Assumption A. $V: \mathbf{C}^{n} \rightarrow \mathbf{C}$ is a holomorphic function such that:

$$
V(q)=\sum_{|\alpha|=2}^{\infty} a_{\alpha} q^{\alpha}, V\left(\mathbb{R}^{n}\right) \subseteq \mathbb{R}_{+} .
$$

(ii) There exist $l_{1}>0, l_{2}>0$ such that $|V(q)| \leqq l_{1} \exp \left(l_{2}|q|^{\frac{1}{n+1}}\right)$ for any $q \in \mathbf{C}^{n}$.

Assumption B. The Schrödinger operator corresponding to $H(p, q, \varepsilon)$, defined as the maximal operator $Q_{\varepsilon}\left(q, D_{q}\right)$ in $L^{2}\left(\mathbf{R}^{n}\right)$ generated by the differential expression

$$
\frac{1}{2}\left(-h^{2} \Delta_{q}+\langle q, q\rangle\right)+\varepsilon V(q)-\frac{n}{2} h
$$

is self-adjoint.

Remarks. (a) All polynomials of even degree with real coefficients and real positive coefficient of the leading term fulfill Assumptions A and B.

(b) The constant $\frac{1}{2} n h$, i.e. the lowest eigenvalue of $Q_{0}$, is subtracted because the unitary image of $Q_{0}$ under $U$ in $\mathscr{F}_{n}$ is just the maximal operator generated by $h\left\langle z, \nabla_{z}\right\rangle$.

The linear complex canonical transformation (1.2) maps (1.10) into $H\left(C^{-1}(z, \bar{z}), \varepsilon\right)=K(z, \bar{z}, \varepsilon)$, where:

$$
K(z, \bar{z}, \varepsilon)=K_{0}(z, \bar{z})+\varepsilon V\left(\frac{z+\bar{z}}{\sqrt{2}}\right),
$$

$K_{0}(z, \bar{z})$ being given by (1.3). Correspondingly, the Bargmann transformation (1.7) yields in $\mathscr{F}_{n}$ the unitary equivalent operators $U Q_{0} U^{-1}=P_{0}$, which is the maximal operator generated by $h\left\langle z, \nabla_{z}\right\rangle$, and $U Q_{\varepsilon} U^{-1}=P_{\varepsilon}$, which is the maximal operator generated by $h\left\langle z, \nabla_{z}\right\rangle+\varepsilon V\left(\frac{z+h \nabla_{z}}{\sqrt{2}}\right)$. We remark that when $V$ is not a polynomial $P_{\varepsilon}$ can be realized as an analytic pseudodifferential operator as discussed in Sect. 2. Given the Hamiltonian (1.11), we denote by $w(t, z,-i \bar{z}, \varepsilon) \equiv w(t, z)$ the flow of the initial condition $(z, \zeta), \zeta=-i \bar{z}$, at time $t$. We further denote by $H_{\varphi_{0}}$ the Sjöstrand space with weight $\varphi_{0}$ (see Definition 2.0 below) and, as usual, by $[x]$ the integer part of $x$. Consider now the Schrödinger initial value problem

$$
\begin{gathered}
\left(i h \partial_{t}-P_{\varepsilon}\left(z, \partial_{z}, h\right)\right) u(t, z)=0 \\
u(t, z)_{\mid t=0}=u_{0}(z)
\end{gathered}
$$

with $u_{0}(z) \in \mathscr{F}_{n}$. Then we have:

Theorem. There are constants $C, C_{1}, C_{2}, C_{3}, C_{4}$, and $B(\varepsilon), T(B(\varepsilon))$ which tend to $+\infty$ as $\varepsilon \downarrow 0$, a weight $\psi_{t}$ and a distribution $u(t, z)$ belonging to the Sjöstrand space $H_{\psi_{t}}$ such that, for $|z| \leqq B,|t| \leqq T, N=\left[C_{1}^{2} h^{-1}\right], N_{1}=\left[C^{2} h^{-1}\right]$ :

1.

$$
u(t, z)_{\mid t=0}=u_{0}(z) \text {. }
$$

$$
\left|e^{-h^{-1} \psi_{t}(z)}\left(i h \partial_{t}-P_{\varepsilon}\left(z, \partial_{z}, h\right)\right) u(t, z)\right| \leqq C_{2} h^{N_{1}} \text {. }
$$


3. For any fixed $(z, t) \in B \times[-T, T]$ one has:

$$
\begin{aligned}
u(t, z)= & e^{i h^{-1} S(t, z,-i \bar{w}(t, z))-h^{-1}|w(t, z)|^{2}} u_{0}(w(t, z)) a_{0}(t, z, 0) \\
& +\sum_{l=1}^{N-1} h^{l} \sum_{j=0}^{N_{1}} h^{j} M_{l}\left(a_{j}(t, z,-i \bar{w}(t, z))\right)+R\left(t, z, h^{-1}\right),
\end{aligned}
$$

where $M_{l}\left(a_{j}\right)$ 's are linear combinations of derivatives of the $a_{j}$ 's whose coefficients are derivatives of $S$ and $u_{0}$, all calculated at $-i \bar{w}(t, z)$, and

$$
\left|e^{-h^{-1} \psi_{t}(z)} R\left(t, z, h^{-1}\right)\right| \leqq C_{3} e^{-C_{4} h^{-1}} .
$$

Here $S(t, z, \xi, \varepsilon)$ is the solution of the Hamilton-Jacobi initial value problem:

$$
\begin{gathered}
\partial_{t} S+\left\langle z, i \nabla_{z} S\right\rangle+\varepsilon V\left(\frac{z+i \nabla_{z} S}{\sqrt{2}}\right)=0, \\
S_{\mid t=0}=\langle z, \xi\rangle,
\end{gathered}
$$

the coefficients $a_{j}, j=0,1, \ldots$ are the solutions of the transport equations:

$$
\begin{gathered}
i \partial_{t} a_{0}-\left\langle\left(z+\frac{\varepsilon}{\sqrt{2}} \nabla_{q} V\right), \nabla_{z} a_{0}\right\rangle+\varepsilon\left(\frac{i}{2} A_{2}^{S}+R_{1}\right) a_{0}=0, \\
a_{0 \mid t=0}=1 \\
i \partial_{t} a_{j}-\left\langle\left(z+\frac{\varepsilon}{\sqrt{2}} \nabla_{q} V\right), \nabla_{z} a_{j}\right\rangle+\varepsilon\left(\frac{i}{2} A_{2}^{S}+R_{1}\right) a_{j}=B_{j}\left(a_{0}, \ldots, a_{j-1}\right), \\
a_{j \mid t=0}=0, \\
A_{2}^{S}(z, \xi)=\sum_{i, j=1}^{n} V_{i j} \partial_{z_{i}, z_{j}}^{2} S, V_{i j}=\left.\partial_{\bar{w}_{i} \bar{w}_{j}}^{2} V\left(\frac{z+i\left(\nabla_{z} S\right)(t, z, \xi)-\bar{w}}{\sqrt{2}}\right)\right|_{\bar{w}=0} .
\end{gathered}
$$

$R_{1}$ is the 2-nd term of the symbol of $P_{\varepsilon}$ realized as an analytic pseudodifferential operator and $B_{j}\left(a_{0}, \ldots, a_{j-1}\right)$ is a linear expression of derivatives of $a_{j}$ 's.

Remarks. (i) According to the standard sign convention in writing the timedependent Schrödinger equation $u(t, z)$ is a local approximation of $\left(e^{-i h^{-1} t K} u_{0}\right)(z)$, so that the classical flow is actually the backward one: $u(t, z(t, w))=u_{0}(w)$.

(ii) Since the integers $N$ and $N_{1}$ tend to infinity as $h$ tends to zero, $u(t, z)$ is actually, by (2), a local solution in the Sjöstrand space $\bar{H}_{\psi_{t}}$ modulo $h^{\infty}$. In this sense, i.e. up to terms $O\left(h^{\infty}\right)$ included in $R\left(t, z, h^{-1}\right), a_{0}(t, z, 0)$ represents the sum of the series

$$
\sum_{|\alpha|=0}^{N-1} \frac{1}{\alpha !} \partial_{\xi}^{\alpha} a_{0}(t, z,-i \bar{w}(t, z))(i \bar{w}(t, z))^{\alpha}
$$

which arises by a direct application of the analytic stationary phase expansion.

(iii) For $\varepsilon=0$ (harmonic case) we have $a_{0}=1, a_{j}=0, j=1, \ldots ; S(t, z, \xi)$ $=\langle z, \xi\rangle e^{i t}, w(t, z)=z e^{-i t}$. Since $\xi=-i \bar{w}(1.13)$ reduces to $u(t, z)=u_{0}\left(z e^{-i t}\right)$, which is the formula obtained above by direct integration of the Schrödinger equation. 
(iv) Formula (1.13) should be compared, e.g., with formula (0.7) of [Ya 1]. Note in this connection that the Maslov indices do not appear here because our solution is local both in space and time, that $a_{0}(t, z, 0)$ (see below) is nothing else but the square root of the absolute value of the Jacobian determinant of the transformation $z \mapsto w(t, z)$, and that the action (0.9) of [Ya1] is in the present case $S(t, z, \xi)$.

(v) To obtain the most explicit representation in terms of the classical motions, [formula (1.13) above] we will avoid the introduction of the Morse coordinates around the critical point, because they are not explicitly known a priori. Instead we will work out the phase in such a way to obtain a quadratic critical point, so that (1.13) will follow by a direct application of the analytic stationary phase expansion $[\mathrm{Sj}$, p. 14] plus an estimate of the remainder.

\section{Proof of the Theorem}

The basic idea of the proof will be to work out in the complexified phase space $\mathbf{C}^{2 n}$ $\cong T_{(1,0)}^{*} \mathbf{C}^{n}$ (the holomorphic cotangent bundle of $\mathbf{C}^{2 n}$, see [N]), where it will be possible to realize $P_{\varepsilon}$ as an analytic pseudodifferential operator in the sense of Sjöstrand and to construct a solution of the initial value problem (1.13) by means of a suitable "localized" parametrix under the form of a Fourier Integral Operator with complex phase.

More precisely, given $\left(u(w)\right.$ in a suitable Sjöstrand space $H_{\psi}$ (see Definition 2.0 below), we will construct, (following e.g. [GeSj, $\mathrm{GrSj}, \mathrm{LSj}]$ ) a phase $S(t, z, \xi ; \varepsilon)$, "transport coefficients" $a_{j}(t, z, \xi ; \varepsilon)$ and determine a contour $\Gamma_{0}$ in $\mathbf{C}^{2 n}$ such that, for all $N \in \mathbf{N}$,

$$
E(t) u(z)=(2 \pi h)^{-n} \int_{\Gamma_{0}} e^{i h^{-1}(S(t, z, \xi ; \varepsilon)-\langle w, \xi\rangle)} \sum_{j=0}^{N} a_{j}(t, z, \xi ; \varepsilon) h^{j} u(w) d w \wedge d \xi
$$

is locally in $H_{\psi_{t}}$ and:

$$
\begin{gathered}
e^{-h^{-1} \psi_{t}(z)}\left(i h \partial_{t}-P_{\varepsilon}\left(z, \partial_{z}, h\right)\right) E(t) u(z)=O\left(h^{N}\right) . \\
E(0) u=u .
\end{gathered}
$$

[We recall that Eqs. (1.15-17) are obtained by looking for a solution of the form (2.1), inserting in (1.12) and requiring the vanishing of all resulting powers of $h$.] $E(t)$ will therefore be the "localized" parametrix, yielding $u(t, z)=E(t) u(z)$. The representation (1.13) is then to be obtained by application of the analytic stationary phase expansion [Sj, p. 14]; in this particular point our treatment will not follow the general theory of [Sj]; in fact, as already mentioned in Remark (iv) above, we will avoid the introduction of the Morse coordinates by reelaborating the phase in such a way to generate a quadratic term, which will be possible for $\varepsilon$ suitably small.

Let us begin by making more explicit the above remark that the linear complex canonical transformation (1.2) can be holomorphically continued to a canonical map of $\mathbf{C}^{2 n}$ onto itself. Consider indeed $x \in \mathbf{C}^{n}, y \in \mathbf{C}^{n}$ with $\operatorname{Re}(x)=q, \operatorname{Re}(y)=p$, and set, for $z \in \mathbf{C}^{n}$ :

$$
\tilde{\varphi}(x, z)=\frac{i}{2}(\langle z, z\rangle+\langle x, x\rangle-2 \sqrt{2}\langle z, x\rangle)
$$


[that is $\tilde{\varphi}$ is the holomorphic continuation of the Bargmann phase (1.6)] and let $\tilde{\chi}_{\varphi}$ be the bijection of $\mathbf{C}^{2 n}$ onto itself defined as

$$
\tilde{\chi}_{\varphi}:\left(x,-\partial_{x} \tilde{\varphi}(x, z) \equiv y=-i(x-\sqrt{2} z)\right) \mapsto\left(z, \partial_{z} \tilde{\varphi}(x, z) \equiv \zeta=i(z-\sqrt{2} x)\right),
$$

whence:

$$
z=\frac{1}{\sqrt{2}}(x-i y), i \zeta=\frac{1}{\sqrt{2}}(x+i y)
$$

for $x, y \in \mathbf{C}^{n}$. When $(x, y)=(q, p) \in \mathbf{R}^{2 n}$ then $\tilde{\varphi}=\varphi, \tilde{\chi}_{\varphi}=\chi_{\varphi}$ and $\zeta=-i \bar{z}$. Hence $\tilde{\chi}_{\varphi}\left(T^{*} \mathbf{R}^{n}\right)=\Lambda=\left\{(z, \zeta) \in \mathbf{C}^{2 n} ; \zeta=-i \bar{z}\right\}$. The F.B.I. theory of Sjöstrand [Sj] requires the examination of the critical points (in $q$ ) of $-\operatorname{Im} \varphi(z, q)$. We immediately have:

$$
\text { c.v.q } \varphi(z, q) \equiv \varphi_{0}(z)=-\operatorname{Im} \varphi(z, q(z))=\frac{1}{2}|z|^{2} ; \quad \text { here } \quad q(z)=\sqrt{2} \operatorname{Re}(z) .
$$

In this case we reobtain the Bargmann phase $\varphi_{0}$, and the manifold $\Lambda$ can be further characterized as the real analytic submanifold of $T_{(1,0)}^{*} \mathbf{C}^{n}$ defined as $\Lambda=\left\{(z, \zeta) \in \mathbf{C}^{2 n} ; \zeta=\frac{2}{i} \nabla_{z} \varphi_{0}\right\}$. Now consider the $(2,0)$-forms in $\mathbf{C}^{2 n}$,

$$
\tilde{\omega}=\sum_{j=1}^{n} d y_{j} \wedge d x_{j} ; \tilde{\sigma}=\sum_{j=1}^{n} d \zeta_{j} \wedge d z_{j},
$$

and their restrictions to $T^{*} \mathbf{R}^{n}$ and $\Lambda$, respectively:

$$
\omega=\tilde{\omega}_{\mid T^{* \mathbf{R}^{n}}}=\sum_{j=1}^{n} d p_{j} \wedge d q_{j} ; \sigma=\tilde{\sigma}_{\mid \Lambda}=\frac{2}{i} \bar{\partial} \partial \varphi_{0},
$$

where as usual $\bar{\partial} f=\sum_{k=0}^{n} \partial_{\bar{z}_{k}} f d \bar{z}_{k}$, and analogous definition for $\partial f$. Then it is easy to check that $\tilde{\chi}_{\varphi}^{*}(\tilde{\sigma})=\tilde{\omega}$ and $\chi_{\varphi}^{*}(\sigma)=\omega$, i.e. the transformations $\tilde{\chi}_{\varphi}$ and $\chi_{\varphi}$ are canonical. In this way the Hamiltonian $H(x, y, \varepsilon)=\frac{1}{2}(\langle x, x\rangle+\langle y, y\rangle+\varepsilon V(x))$, which is the holomorphic continuation of (1.10) to $\mathbf{C}^{2 n}$, has canonical image under $\tilde{\chi}_{\varphi}$ given by:

$$
K(z, \zeta, \varepsilon) \equiv H\left(\tilde{\chi}_{\varphi}^{-1}(z, \zeta), \varepsilon\right)=\langle z, i \zeta\rangle+\varepsilon V\left(\frac{z+i \zeta}{\sqrt{2}}\right) .
$$

In turn $K(z, \zeta, \varepsilon)$ is nothing else than the holomorphic continuation of $K(z, \bar{z}, \varepsilon)$, the image of $H(p, q, \varepsilon)$ under $\chi_{\varphi}$ :

$$
K(z, \bar{z}, \varepsilon)=K(z, \zeta, \varepsilon)_{\mid \Lambda}=|z|^{2}+\varepsilon V\left(\frac{z+\bar{z}}{\sqrt{2}}\right) .
$$

Therefore $\Lambda$ is by construction an invariant manifold of the Hamiltonian vector field associated with $K(z, \zeta, \varepsilon)$ : the restriction to $\Lambda$ of the flow in $\mathbf{C}^{2 n}$ generated by (2.6) is of course the flow generated by $K(z, \bar{z}, \varepsilon)=|z|^{2}+\varepsilon V\left(\frac{z+\bar{z}}{\sqrt{2}}\right)$. We shall henceforth freely use, without any further specification, this possibility of looking at the given Hamiltonian flow as the (invariant) restriction of the flow of the 
holomorphic Hamiltonian field on $\mathbf{C}^{2 n}$,

$$
\sum_{j=1}^{n}\left(\frac{\partial K}{\partial \zeta_{j}} \frac{\partial}{\partial z_{j}}-\frac{\partial K}{\partial z_{j}} \frac{\partial}{\partial \zeta_{j}}\right)
$$

The first step in the construction of the local solution of the Schrödinger initial value problem by means of the technique of the FIO on complex domains is represented by the realization of $P_{\varepsilon}$ as an analytic pseudodifferential operator in the sense of Sjöstrand. First let us recall the definition of the Sjöstrand space $H_{\phi}^{\text {loc }}(\Omega)$, where $\Omega$ is an open subset of $\mathbf{C}^{n}$, and $\phi: \Omega \rightarrow \mathbf{R}_{+}$is continuous.

2.0. Definition. Let $u(z, h)$ be a holomorphic function on $\Omega$ for any $h>0$. Then $u \in H_{\phi}^{\text {loc }}(\Omega)$ iff for any $K \Subset \Omega$ and for any $\varepsilon>0$ there is $C_{\varepsilon}$ such that $|u(z, h)|$ $\leqq C_{\varepsilon} e^{h^{-1}(\phi(z)+\varepsilon)}$ for any $z \in K$, any $\left.h \in\right] 0,1\left[\right.$. For any given $x_{0} \in \Omega$ the space $H_{\phi, x_{0}}$ is defined by the equivalence classes of functions $u^{\prime}$ defined as follows: if $(u, v) \in H_{\phi}^{\text {loc }}(\Omega)$ we shall say that $u \approx v$ ( $u$ equivalent to $v$ ) on $x_{0}$ if $u-v \in H_{\phi^{\prime}}^{\text {loc }}(W)$ in a neighborhood $W$ of $x_{0}$ and some $\phi^{\prime}<\phi$.

In our case $x_{0}$ will be the origin in $\mathbf{C}^{n}$ and our operator will act in the spaces $H_{\phi, x_{0}}$. Notice also that $\mathscr{F}_{n} \subset H_{\varphi_{0}}\left(\mathbf{C}^{n}\right)$, where $\varphi_{0}(z)=\frac{1}{2}|z|^{2}$ is the Bargmann phase.

2.1. Lemma. Let

$$
a(z, \zeta, h)=e^{-i h^{-1}\langle z, \zeta\rangle} P_{\varepsilon}\left(z, \partial_{z}, h\right) e^{i h^{-1}\langle\cdot, \zeta\rangle}
$$

be the symbol of $P_{\varepsilon}$ as a pseudodifferential operator in $\mathbf{C}^{2 n}$. Then a $(z, \zeta, h)$ is a formal analytic symbol in the sense of Sjöstrand, with:

$$
a(z, \zeta, h)=p_{0}(z, \zeta, \varepsilon)+\varepsilon \sum_{j=1}^{\infty} R_{j}(z, i \zeta) h^{j}
$$

where the principal symbol $p_{0}$ is the classical Hamiltonian,

$$
p_{0}(z, \zeta, \varepsilon)=\langle z, i \zeta\rangle+\varepsilon V\left(\frac{z+i \zeta}{\sqrt{2}}\right)
$$

and:

$$
R_{j}(z, i \zeta)=\sum_{|t|=j+\mid 1}^{2 j}\left(\frac{1}{\sqrt{2}}\right)^{|t|}\left(\partial_{q}^{t} V\right)\left(\frac{z+i \zeta}{\sqrt{2}}\right) \sum_{2 \leqq|\mu| \leqq j+1}^{*} \frac{1}{a_{\mu} !}\left(\frac{1}{\sqrt{2} \mu !} \partial_{z}^{\mu}\left(\left\langle z, \frac{z}{2}+i \zeta\right\rangle\right)\right)^{a_{\mu}} .
$$

Here $\sum^{*}$ is the sum over all non-negative integers $a_{\mu}$ such that

$$
\sum_{2 \leqq|\mu| \leqq j+1} a_{\mu}=|t|-j, \sum_{2 \leqq|\mu| \leqq j+1} \mu_{i} a_{\mu}=t_{i}, \quad i=1,2, \ldots, n,
$$

and for any compact subset $\Omega=\Omega_{1} \times \Omega_{2}$ of $\mathbf{C}^{n}$ there are constants $k_{1}$ and $k_{2}$ such that

$$
\max _{(z, \zeta) \in \Omega_{1} \times \Omega_{2}}\left|R_{j}(z, i \zeta)\right| \leqq k_{1} k_{2}^{j}
$$

Proof. The proof of (2.8), (2.9), (2.10) is identical to that of Lemma 2.4 of [GrPa] and can therefore be omitted. The estimate $(2.10)$ implies that $a(z, \zeta, h)$ is a formal analytic symbol $([\mathrm{Sj}])$. 
Remark. By this lemma we can thus write the formal pseudodifferential representation:

$$
P_{\varepsilon}\left(z, \partial_{z}, h\right) u(z)=(2 \pi h)^{-n} \int_{\Gamma} e^{i h^{-1}\langle z-w, \xi\rangle} a(z, \xi, h) u(w) d w \wedge d \xi
$$

using a representative of $a\left(\right.$ i.e., a suitable $N=N(h)$ in the sum $\left.\sum_{j=0}^{N} R_{j} h^{j}\right)$. We will see below how to specify a suitable integration contour $\Gamma$ in $\mathbf{C}^{2 n}$ ("good contour" in the terminology of Sjöstrand [Sj]) which makes $P_{\varepsilon}\left(z, \partial_{z}, h\right)$ a well defined pseudodifferential operator in a suitable space $H_{\phi}$.

We now proceed to the construction of the phase $S$ in the standard way, i.e. by the solutions of the Hamilton's equations (2.12) below and Hamilton-Jacobi theory; since however we are working in complex phase space, the positivity properties which immediately ensure global existence of the Hamilton flow and the invariance of suitable open sets under it do not hold so that these questions have to be preliminarily examined.

Consider the Hamilton equations in $\mathbf{C}^{2 n}$ :

$$
\left.\begin{array}{c}
\frac{d z}{d t}=\frac{\partial K}{\partial \zeta}(z, \zeta, \varepsilon)=i z+i \frac{\varepsilon}{\sqrt{2}}\left(\partial_{q} V\right)\left(\frac{z+i \zeta}{\sqrt{2}}\right), z(0, w, \xi)=w \\
\frac{d \zeta}{d t}=-\frac{\partial K}{\partial z}(z, \zeta, \varepsilon)=-i \zeta-\frac{\varepsilon}{\sqrt{2}}\left(\partial_{q} V\right)\left(\frac{z+i \zeta}{\sqrt{2}}\right), \zeta(0, w, \xi)=\xi .
\end{array}\right\}
$$

Since $K(z, \zeta, \varepsilon)$ is real analytic in $(t, \varepsilon) \in \mathbf{R}^{2}$, and holomorphic in $(z, \zeta) \in \mathbf{C}^{2 n}$, the mappings $(t, w, \xi, \varepsilon) \mapsto(z, \zeta)$ are holomorphic in the $(w, \xi)$ variables and real analytic in the $(t, \varepsilon)$ variables (this follows from a compactness argument if $(t, w, \xi, \varepsilon)$ belong to compact sets; see e.g. [N]), the domain of definition being specified in Lemma 2.3 below. Moreover let us note, for further convenience, that we can write:

$$
\begin{aligned}
& z(t, w, \xi, \varepsilon)=z(t, w, \xi, 0)+\varepsilon \int_{0}^{1}\left(\partial_{\varepsilon} z\right)(t, w, \xi, \tau \varepsilon) d \tau=w e^{i t}+\varepsilon I_{1}(t, w, \xi, \varepsilon) \\
& \zeta(t, w, \xi, \varepsilon)=\zeta(t, w, \xi, 0)+\varepsilon \int_{0}^{1}\left(\partial_{\varepsilon} \zeta\right)(t, w, \xi, \tau \varepsilon) d \tau=\xi e^{-i t}+I_{2}(t, w, \xi, \varepsilon)
\end{aligned}
$$

with $\varepsilon\left|I_{k}(t, w, \xi, \varepsilon)\right| \rightarrow 0$ as $\varepsilon \rightarrow 0, k=1,2 ;(t, w, \xi)$ fixed and, once chosen a suitable compact set in $(t, w, \xi, \varepsilon), \varepsilon \operatorname{Max}\left|I_{k}\right| \rightarrow 0$ as $\varepsilon \rightarrow 0$.

2.2. Lemma. Let $M$ be a manifold (differentiable, analytic, holomorphic), $K$ a compact subset of $M$ and $v$ a vector field on $M$ of class $C^{r}\left(C^{\infty}, C^{\omega}\right.$, holomorphic). For any $p \in M$ let $\varphi(t, p)$ be the flow of $v$, and let $D$ be a compact subset of $K$ with $D \neq K$ and $D \cap \partial K=\emptyset$. Then if $p \in D$ there is $T>0$ such that $\varphi(]-T, T[\times D) \subset K$.

Proof. Let us first remark that it is well known that the flow has locally the same regularity as $M$ and $v$. By [A, Corollary 5] if $p \in K$ then either $\varphi(t, p)$ exists for all 
$t \in \mathbf{R}$ or there is $T_{p}>0$ such that $\varphi(t, p)$ is defined for $|t| \leqq T_{p}$ and $\varphi\left(T_{p}, p\right)$ [or $\left.\varphi\left(-T_{p}, p\right)\right]$ belongs to $\partial K$. Without loss, consider only the $t>0$ case. Let

$$
P_{+}=\{T>0 ; \exists p \in D \text { such that } \varphi(t, p) \in K, \forall t: 0 \leqq t \leqq T, \varphi(T, p) \in \partial K\},
$$

and set $T_{+}=\inf P_{+}$. Suppose $T_{+}=0$. Then for any $\varepsilon>0$ there is $p_{\varepsilon} \in D$ such that $\varphi\left(\varepsilon, p_{\varepsilon}\right) \in \partial K$. Choose $\varepsilon_{n}=\frac{1}{n}$. Then for any $n \in \mathbf{N}$ there is $p_{n} \in D$ such that $\varphi\left(\frac{1}{n}, p_{n}\right) \in \partial K$. Since $p_{n} \in D$, and $D$ is compact, there is $\left(n_{k}\right)_{k \in \mathbf{N}}$ such that $p_{n_{k}} \rightarrow p \in D$ and $\frac{1}{n_{k}} \rightarrow 0$ as $k \rightarrow \infty$. By the regularity of the flow and the closedness of $\partial K$ we have $\varphi\left(\frac{1}{n_{k}}, p_{n_{k}}\right) \rightarrow y \in \partial K$. On the other hand $\varphi(0, p)=p \in D$, which contradicts $\partial K \cap D=\emptyset$. This proves Lemma 2.2.

The further property of the complex Hamiltonian flow needed in what follows is contained in the next preliminary result. Fix $0<A_{0}<A_{1}, T\left(\varepsilon_{0}\right) \equiv T, \varepsilon_{0}$ in such a way that $(t, w, \xi, \varepsilon) \mapsto(z, \zeta)$ is regular [i.e., holomorphic with respect to $(w, \xi)$ and real analytic with respect to $(t, \varepsilon)]$ for $(t, w, \xi, \varepsilon) \in[-T, T] \times B_{0} \times B_{0} \times\left[0, \varepsilon_{0}\right] \equiv I(T)$ $\times B_{0} \times B_{0} \times I\left(\varepsilon_{0}\right)$ where $B_{i}=\left\{z \in \mathbf{C}^{n} ;|z| \leqq A_{i}\right\}, i=0,1$.

2.3. Lemma. There are $\varepsilon_{1}$ and $A, 0<\varepsilon_{1}<\varepsilon_{0}, 0<A<A_{0}$ such that:

(i) The mapping $w \mapsto z(t, w, \xi, \varepsilon)$ is, for any fixed $(t, \xi, \varepsilon) \in I(T) \times B_{0} \times I\left(\varepsilon_{1}\right), a$ holomorphic diffeomorphism on $B_{0}$;

(ii) Let $B=\left\{z \in \mathbf{C}^{n} ;|z| \leqq A\right\}$. Then $z\left(t, B_{0}, \xi, \varepsilon\right) \subset B$ for any $(t, \xi, \varepsilon) \in I(T)$ $\times B_{0} \times I\left(\varepsilon_{1}\right)$.

Proof. Set: $K=B_{1} \times B_{1} ; D=B_{0} \times B_{0}$, and denote once again by $T$ the minimum between the $T$ of Lemma 2.2 and $T\left(\varepsilon_{0}\right)$ defined above. Therefore there are constants $C_{i}\left(\varepsilon_{0}\right), i=1,2$, such that:

$$
\max _{I(T) \times B_{1} \times B_{1} \times I\left(\varepsilon_{0}\right)}\left|\frac{\partial I_{i}}{\partial w}(t, w, \xi, \varepsilon)\right| \leqq C_{i}\left(\varepsilon_{0}\right) .
$$

Since $\frac{\partial z}{\partial w}=e^{i t} I_{n \times n}+\varepsilon \frac{\partial I_{i}}{\partial w}(t, w, \xi, \varepsilon), z(t, w, \xi, \varepsilon)$ is invertible with respect to $w$ for $\varepsilon<\varepsilon_{1}$ on $I(T) \times B_{0} \times B_{1} \times I\left(\varepsilon_{0}\right)$ where $\varepsilon_{1}<\frac{1}{C_{1}}$ is fixed. Now $z(t, w, \xi, \varepsilon)=w e^{i t}$ $+\varepsilon a(t, w, \xi, \varepsilon), \zeta(t, w, \xi, \varepsilon)=\zeta e^{-i t}+\varepsilon b(t, w, \xi, \varepsilon)$. Let:

$$
\begin{aligned}
M(a) & =\max _{I(T) \times B_{0} \times B_{0} \times I\left(\varepsilon_{0}\right)}|a(t, w, \xi, \varepsilon)|, \\
M(b) & =\max _{I(T) \times B_{0} \times B_{0} \times I\left(\varepsilon_{0}\right)}|b(t, w, \xi, \varepsilon)|, \\
M(a, b) & =\max \{M(a), M(b)\} .
\end{aligned}
$$

Then it is enough to choose $\varepsilon_{1}$ in such a way that $A=A_{0}-\varepsilon_{1} M(a, b)>0$ in order to obtain, with $B=\left\{z \in \mathbf{C}^{n} ;|z| \leqq A\right\}, z\left(t, B_{0}, \xi, \varepsilon\right) \subset B$ for any $(t, \xi, \varepsilon) \in I(T) \times B_{0} \times I\left(\varepsilon_{0}\right)$. Moreover we can also conclude that $\zeta\left(t, w, B_{0}, \varepsilon\right) \subset B$ for any $(t, \xi, \varepsilon) \in I(T)$ $\times B_{0} \times I\left(\varepsilon_{1}\right)$. This proves Lemma 2.3 . 
After these preliminary results we are ready to solve the Hamilton-Jacobi and the transport equations. We have:

2.4. Proposition. Equations (1.15-17) admit a solution $S(t, z, \xi, \varepsilon), a_{j}(t, z, \xi, \varepsilon)$, respectively, which are holomorphic functions of $(z, \zeta) \in B \times B_{0}$, and real analytic functions of $(t, \varepsilon) \in I(T) \times I\left(\varepsilon_{1}\right)$. Furthermore $\sum_{j \geqq 0} a_{j} h^{j}$ is a formal analytic symbol in the sense of Sjöstrand, and

$$
\nabla_{\xi} S=w(t, z, \xi, \varepsilon), \quad \nabla_{z} S=\zeta(t, w(t, z, \xi, \varepsilon), \xi, \varepsilon) .
$$

Proof. First remark that (1.15), (1.16), (1.17) can be rewritten as, respectively:

$$
\left.\left.\begin{array}{c}
\partial_{t} S+K\left(z, \nabla_{z} S, \varepsilon\right)=0, \\
S_{\mid t=0}=\langle z, \xi\rangle,
\end{array}\right\} \begin{array}{c}
\partial_{t} a_{0}+\left\langle i z+i \frac{\varepsilon}{\sqrt{2}} \nabla_{q} V, \nabla_{z} a_{0}\right\rangle+\varepsilon\left(\frac{1}{2} A_{2}^{S}-i R_{1}\right) a_{0}=0 \\
a_{0 \mid t=0}=1, \\
\partial_{t} a_{j}+\left\langle i z+i \frac{\varepsilon}{\sqrt{2}} \nabla_{q} V, \nabla_{z} a_{j}\right\rangle+\varepsilon\left(\frac{1}{2} A_{2}^{S}-i R_{1}\right) a_{j}=f_{j} \\
a_{j \mid t=0}=0,
\end{array}\right\}
$$

with obvious definition of $f_{j}, j=1,2, \ldots$ By the holomorphic analog of the standard Hamilton-Jacobi theory, it is well known that the formula

$$
S(t, z, \xi, \varepsilon)=\langle z, \xi\rangle-\int_{0}^{t} K(z, \zeta(s, w(s, z, \xi, \varepsilon), \xi, \varepsilon), \varepsilon) d s
$$

yields a solution of the initial value problem (2.15) on $I(T) \times B \times B_{0} \times I\left(\varepsilon_{1}\right)$ for any $\varepsilon_{1}<\varepsilon_{0}$.

$S$ is holomorphic in $(z, \xi)$ and real analytic in $(t, \varepsilon)$. Moreover we have:

$$
\begin{gathered}
\nabla_{z} S(t, z, \xi, \varepsilon)=\zeta(t, w(t, z, \xi, \varepsilon), \xi, \varepsilon) \\
\nabla_{\xi} S(t, z, \xi, \varepsilon)=w(t, z, \xi, \varepsilon),
\end{gathered}
$$

and we can also write $S(t, z, \xi, \varepsilon)$ under the form:

$$
S(t, z, \xi, \varepsilon)=\langle z, \xi\rangle e^{-i t}+\varepsilon S_{1}(t, z, \xi, \varepsilon),
$$

where $\langle z, \xi\rangle e^{-i t}$ is the phase function corresponding to the unperturbed flow generated by $K_{0}$. Given $S,(2.16)$ and (2.17) can be solved by the well known solution technique for first order partial differential equations. The result is:

$$
\begin{aligned}
& a_{0}(t, z, \xi, \varepsilon)=\exp \left(\varepsilon \int_{0}^{t}\left(i R_{1}(s, z, \xi)-\frac{1}{2} A_{2}^{S}(s, z, \xi)\right)_{\mid z=z(s, w(t, z, \xi), \xi)} d s\right) \\
& a_{j}(t, z, \xi, \varepsilon)=a_{0}(t, z, \xi, \varepsilon) \int_{0}^{t} \tilde{f}_{j}(s, t, z, \xi) \\
& \quad \times\left(\exp \left(-\varepsilon \int_{0}^{s}\left(i R_{1}(\tau, z, \xi)-\frac{1}{2} A_{2}^{S}(\tau, z, \xi)\right)_{\mid z=z(\tau, w(s, z, \xi), \xi)} d \tau\right) d s\right),
\end{aligned}
$$


where $f_{j}(s, t, z, \xi)=f_{j}(s, z(s, w(t, z, \xi), \xi), \xi)$. These functions are holomorphic with respect to $(z, \xi) \in B \times B_{0}$ and real analytic with respect to $(t, \varepsilon) \in I(T) \times I\left(\varepsilon_{1}\right)$. For $\varepsilon=0$ we have $a_{0}=1, a_{j}=0$ for all $j>0$. Consider now:

$$
e^{i h^{-1} S} \sum_{j=0}^{N} a_{j} h^{j} \equiv e^{i h^{-1}} a_{N}
$$

Let us verify that $a_{\infty}$ is a formal analytic symbol by using [Sj, Theorem 9.3]. In the same notation, set indeed $p(t, z, w ; \tau, \zeta, \zeta)=-\tau-p_{0}(z, \zeta)$ and

$$
(x, \eta)=((t, z, w) ;(\tau, \zeta, \xi)) \in \mathbf{C}^{2 n+1} \times \mathbf{C}^{2 n+1},\left(x_{0}, \eta_{0}\right)=(0,0), H:=\{t=0\}
$$

(that is, $H$ is the initial hyperplane $t=0$ in $\mathbf{C}^{2 n+1}$ ). We have:

$$
\frac{\partial p}{\partial \tau}=-1 \neq 0, p(0)=0, \quad \text { and } \quad \nabla_{(\tau, \zeta, \xi)} p=\left(-1, \nabla_{(\zeta, \xi)} p_{0}\right)=(-1, v)
$$

Now $(-1, v)$ is obviously transverse to $H$, and

$$
\nabla_{(t, z, \xi)} S(0,0,0)=0=\eta_{0}, p\left(t, z, \xi ; \nabla_{(t, z)} S\right)=0 .
$$

Hence the equation

$$
\begin{gathered}
e^{-i h^{-1} S}\left(i h \partial_{t}-P_{\varepsilon}\left(z, \partial_{z}, h\right)\right) e^{i h^{-1} S} a=0, \\
a_{\mid t=0}=1,
\end{gathered}
$$

where $a=\sum_{j} \tilde{a}_{j} h^{j}$, has a solution $a_{\infty}$ which is a formal analytic symbol by $[\mathrm{Sj}$, Theorem 9.3]. Let us now prove that, modulo terms exponentially vanishing in $h^{-1}, a=a_{\infty}$. To this end set, for $|z| \leqq \frac{A}{3},|z-w| \leqq \frac{A}{2}$ (so that $z-w \in B$ ) and $(t, \varepsilon) \in I(T) \times I\left(\varepsilon_{1}\right)$ :

$$
\Gamma_{z, \xi, t}=\left\{(w, \theta) \in \mathbf{C}^{2 n} ; \theta=\nabla_{z} S(t, z, \xi)+i R(\overline{z-w}),|z-w| \leqq \frac{A}{2}\right\},
$$

where $R$ is a constant greater than the maximum of the Hessian matrix of $S$ over $I(T) \times B_{0} \times B_{0} \times I\left(\varepsilon_{1}\right)$. It will be seen below that $\Gamma_{t, z, \xi}$ is a "good contour" for (2.11); if this holds, we can write

$$
\begin{aligned}
A_{\varepsilon}(z, \xi, t) \equiv P_{\varepsilon}\left(z, \partial_{z} ; h\right)\left(e^{i h^{-1} S(t, \cdot, \xi)} \sum_{j=1}^{N_{1}} a_{j}(t, \cdot, \xi) h^{j}\right) \\
=(2 \pi h)^{-n} \int_{\Gamma_{z}, \xi, t} e^{i h^{-1}\langle z-w, \theta\rangle}\left(\langle z, i \theta\rangle+\varepsilon V\left(\frac{z+i \theta}{\sqrt{2}}\right)+\varepsilon \sum_{j=0}^{N_{1}} R_{j}(z, i \theta) h^{j}\right) \\
\quad \times\left(e^{i h^{-1} S(t, w, \xi)} a_{N_{1}}(t, w, \xi)\right) d w \wedge d \theta .
\end{aligned}
$$

By the analytic stationary phase expansion (see [Sj, Example 2.6]) applied to (2.26), the phase being $\langle z-w, \theta\rangle+S(t, w, \xi)$ and the stationary point $z=w$, 
$\theta=\nabla_{z} S(t, z, \xi)$ we can therefore write, with $C=\frac{A}{2}$ :

$$
\begin{aligned}
A_{\varepsilon}(z, \xi, t)=(2 \pi h)^{-n} \int_{|z-w| \leqq C} e^{-R h^{-1}|z-w|^{2}}\left(\left\langle z, i \nabla_{z} S-R(\overline{z-w})\right\rangle\right. \\
\left.\quad+\varepsilon V\left(\frac{z+i \nabla_{z} S-R(\overline{z-w)}}{\sqrt{2}}\right)+\varepsilon \sum_{j=1}^{\infty} R_{j}\left(z, i \nabla_{z} S-R(\overline{z-w})\right) h^{j}\right) \\
\quad \times\left(e^{i h^{-1}\left(S(t, w, \xi)+\left\langle z-w, \nabla_{z} S\right\rangle\right)} a_{N-1}(t, w, \xi)\right) L(d w) \\
=\sum_{|\alpha|=0}^{N_{1}-1} \frac{1}{\alpha !}\left(\frac{h}{R}\right)^{|\alpha|} \partial_{w^{\prime}}^{\alpha} \partial_{\bar{w}^{\prime}}^{\alpha}\left(\left(\left\langle z, i \nabla_{z} S-R \bar{w}^{\prime}\right\rangle+\varepsilon V\left(\frac{z+i \nabla_{z} S-R \bar{w}^{\prime}}{\sqrt{2}}\right)\right.\right. \\
\left.\quad+\varepsilon \sum_{j=1}^{\infty} R_{j}\left(z, i \nabla_{z} S-R \bar{w}^{\prime}\right) h^{j}\right) \\
\left.\quad \times\left(e^{i h^{-1}\left(S\left(t, z-w^{\prime}, \xi\right)+\left\langle w^{\prime}, \nabla_{z} S\right\rangle\right)} a_{N_{1}}\left(t, z-w^{\prime}, \xi\right)\right)\right)_{\mid w^{\prime}=0}+R_{N_{1}}\left(h^{-1}, t, z, \xi\right),(2.2)
\end{aligned}
$$

where the substitution $z-w=w^{\prime}$ has been performed in the integral and, choosing $N_{1}=\left[C^{2} h^{-1}\right]$ :

$$
\left|R_{N_{1}}\left(h^{-1}, t, z, \xi\right)\right| \leqq C(n) e^{-C h^{-1}} \max _{w^{\prime}}\left|e^{i h^{-1}\left(S\left(t, z-w^{\prime}, \xi\right)+\left\langle w^{\prime}, \nabla_{z} S\right\rangle\right.}\right| .
$$

By expanding in Taylor series up to the second order in $w^{\prime}$ with initial point 0 we have:

$$
\begin{aligned}
& -\operatorname{Im}\left(S\left(t, z-w^{\prime}, \xi\right)+\left\langle w^{\prime}, \nabla_{z} S(t, z, \xi)\right\rangle\right) \\
& \quad=-\operatorname{Im}\left(S(t, z, \xi)+\frac{1}{2}\left\langle Q(t, z, \xi, \varepsilon) w^{\prime}, w^{\prime}\right\rangle\right) \leqq-\operatorname{Im} S(t, z, \xi)+C(\varepsilon)\left|w^{\prime}\right|^{2},
\end{aligned}
$$

and choosing $\varepsilon$ in such a way that $C(\varepsilon)-1<0$ we get, since $\left|w^{\prime}\right| \leqq C$ :

$$
\left|R_{N_{1}}\left(h^{-1}, t, z, \xi\right)\right| \leqq C(n) e^{-h^{-1} \operatorname{Im} S(t, z, \xi)} e^{-h^{-1} C(1-C(\varepsilon))},
$$

where the constant $C$ will be specified later. If all coefficients of the power of $h$ are required to vanish we obtain Eqs. (1.15-17). This concludes the proof of Proposition 2.4, modulo the proof of the following

2.5. Lemma. $\Gamma_{z, t, \xi}$ is a good contour for $P_{\varepsilon}$, namely for the phase of the integral (2.26).

Proof. Let us first recall the definition of good contour: the smooth chain $\Gamma$, assumed to be a bijection between $W \Subset \mathbf{R}^{n}$ and $\mathbf{C}^{n}$ together with its differential $d \Gamma$, is a good contour for a smooth real valued function $\phi$ with a saddle point at $x, x \in \Gamma$ if for any $y \in \Gamma$ we have $\phi(y)-\phi(x) \leqq-C|y-x|^{2}$ for some $C>0$. Thus in our case we compute, for $(w, \theta) \in \Gamma_{z, \xi, t}$ :

$$
\begin{aligned}
- & \operatorname{Im}\left(\left\langle z-w, \nabla_{z} S(t, z, \xi)+i R(\overline{z-w})\right\rangle+S(t, w, \xi)\right) \\
& =-\operatorname{Im}\left(S(t, w, \xi)+\left\langle z-w, \nabla_{z} S(t, z, \xi)\right\rangle\right)-R|z-w|^{2} \\
& =-\operatorname{Im} S(t, z, \xi)-\operatorname{Im}\left\langle Q_{t, \xi}(w)(w-z), w-z\right\rangle-R|w-z|^{2} \\
& \leqq-\operatorname{Im} S(t, z, \xi)-(R-C(\varepsilon))|z-w|^{2}
\end{aligned}
$$


so that it is enough to choose $R>C\left(\varepsilon_{1}\right)$ since $C(\varepsilon)<C\left(\varepsilon_{1}\right)$ for $\varepsilon<\varepsilon_{1}$. This proves Lemma 2.5.

Now fix $\gamma>0$ such that $\frac{3}{4}(1+\gamma)^{2}+\frac{1}{2} \gamma(1+\gamma)+C^{\prime}\left(\varepsilon_{1}\right)<1$, where $C^{\prime}\left(\varepsilon_{1}\right)$ $=\max \mid$ Hess $_{\xi} S \mid$. This is always possible if we take $\varepsilon_{1}$ such that $C^{\prime}\left(\varepsilon_{1}\right)<\frac{1}{4}$. Then choose $C_{1}>0$ such that $(1+\gamma) C_{1}<A_{0}$, and $0<C_{2}<A$ in such a way that $|w(t, z)|$ $\leqq \gamma C_{1}$ if $|z| \leqq \frac{C_{2}}{2}$ (shrinking $T$ if necessary). Here $w(t, z)$ is the critical point of

$$
(w, \xi) \mapsto-\operatorname{Im}(S(t, z, \xi, \varepsilon)-\langle w, \xi\rangle)+\varphi_{0}(w),
$$

where $\varphi_{0}(w)=\frac{1}{2}|w|^{2}$. The critical point is given by:

$$
\left.\begin{array}{c}
\nabla_{\xi} S(t, z, \xi, \varepsilon)-w=0 \\
\nabla_{w}(S(t, z, \xi, \varepsilon)-\langle w, \xi\rangle)+\frac{2}{i} \nabla_{w} \varphi_{0}(w)=-\xi-i \bar{w}=0
\end{array}\right\}
$$

whose solution is the classical Hamiltonian flow:

$$
\left.\begin{array}{c}
w=w(t, z,-i \bar{z} ; \varepsilon) \equiv w(t, z ; \varepsilon)=\nabla_{\xi} S(t, z,-i \bar{w}(t, z) ; \varepsilon) \\
\xi=-i \bar{w}(t, z,-i \bar{z} ; \varepsilon) \equiv-i \bar{w}(t, z ; \varepsilon) .
\end{array}\right\}
$$

Notice that $(w, z)$ belongs to $\Lambda$; moreover, since

$$
(w, \xi) \mapsto-\operatorname{Im}(S(t, z, \xi ; 0)-\langle w, \xi\rangle)+\varphi_{0}(w)
$$

has a nondegenerate critical point of signature $(2 n, 2 n)$ in $(0,0)$, the same will be true for (2.28), at least for $\varepsilon_{1}$ suitably small. Therefore the function

$$
\psi_{t}(z)=-\operatorname{Im}(S(t, z,-i \bar{w}(t, z))-\langle w(t, z),-i \bar{w}(t, z)\rangle)+\varphi_{0}(w(t, z))
$$

will be plurisubharmonic, i.e. $\Delta_{z} \psi_{t}(z) \geqq 0$. Moreover:

$$
\left(\nabla_{z} S\right)(t, z,-i \bar{w}(t, z))=\frac{2}{i} \nabla_{z} \psi_{t}(z)
$$

2.6. Lemma. Set: $\Gamma_{0}=\left\{(w, \xi) \in \mathbf{C}^{2 n} ; \xi=-i \bar{w},|w-w(t, z)| \leqq C_{1}\right\}$. Then $\Gamma_{0}$ is a good contour for the phase $-\operatorname{Im}(S(t, z, \xi ; \varepsilon)-\langle w, \xi\rangle)+\frac{1}{2}|w|^{2}$.

Proof. It is easy to check (by polarization) that

$$
-\operatorname{Im}\langle z,-i \bar{w}\rangle \leqq \frac{1}{2}|z|^{2}+\frac{1}{2}|w|^{2}-\frac{1}{4}|z-w|^{2} .
$$

Then, again by Taylor expansion up to second order, this time with respect to $\xi$ :

$$
\begin{aligned}
-\operatorname{Im} & S(t, z,-i \bar{w})-|w|^{2}+\frac{1}{2}|w|^{2} \\
& -\left(-\operatorname{Im} S(t, z,-i \bar{w}(t, z))-|w(t, z)|^{2}+\frac{1}{2}|w(t, z)|^{2}\right) \\
= & -\operatorname{Im}\left(\left\langle\nabla_{\xi} S(t, z,-i \bar{w}(t, z)),-\overline{i(w-w(t, z))}\right\rangle\right. \\
& -\frac{1}{2}\left\langle Q_{t, z}(w)(-\overline{i(w-w(t, z)))},-\overline{i(w-w(t, z))}\rangle\right)-\frac{1}{2}|w|^{2}+\frac{1}{2}|w(t, z)|^{2} \\
= & -\operatorname{Im}\langle w(t, z),-i \bar{w}\rangle-|w(t, z)|^{2} \\
& -\frac{1}{2} \operatorname{Im}\left\langle Q_{t, z}(w) v, v\right\rangle-\frac{1}{2}|w|^{2}+\frac{1}{2}|w(t, z)|^{2} \leqq-\left(\frac{1}{4}-C^{\prime}\left(\varepsilon_{1}\right)\right)|w-w(t, z)|^{2},
\end{aligned}
$$


where $v=-\overline{i(w-w(t, z)}$. Choosing $\varepsilon_{1}$ in such a way that $C^{\prime}\left(\varepsilon_{1}\right)<\frac{1}{4}$ the lemma is proved.

Let us now take $N_{1}=\left[\frac{1}{4} C_{2}^{2} h^{-1}\right]=\left[C_{0}^{2} h^{-1}\right]$ and $N=\left[C_{3}^{2} h^{-1}\right]$, where the constant $C_{3}$ is chosen in such a way that $1<\left(\frac{C_{3}}{C_{1}}\right)^{2}<e$, and

$$
\frac{3}{4}(1+\gamma)^{2}+\frac{1}{2} \gamma(1+\gamma)+C^{\prime}(\varepsilon)<\left(\frac{C_{3}}{C_{1}}\right)^{2}\left(1-\log \left(\frac{C_{3}}{C_{1}}\right)^{2}\right) .
$$

For $u \in \mathscr{F}_{n}$ and $|z| \leqq \frac{C_{2}}{2}$ we can now define:

$$
E(t) u(z)=(2 \pi h)^{-n} \int_{\Gamma_{0}} e^{i h^{-1}(\mathbf{S}(t, z, \xi ; \varepsilon)-\langle w, \xi\rangle)} a_{N_{1}}(t, z, \xi, h) u(w) d w \wedge d \xi .
$$

Then, by Lemma 2.6, $E(t): H_{\varphi_{0}} \rightarrow H_{\psi_{t}, 0}$. Furthermore, let us realize $P_{\varepsilon}$ by choosing the contour

$$
\Gamma_{1}=\left\{(w, \theta) \in \mathbf{C}^{2 n} ; \theta=\frac{2}{i} \nabla_{z} \psi_{t}(z)+i R(\overline{z-w}),|z-w| \leqq \frac{1}{2} C_{2},|z| \leqq \frac{1}{2} C_{2}\right\}
$$

(note that if $|z| \leqq \frac{1}{2} C_{2}$ and $|w-z| \leqq \frac{1}{2} C_{2}$ then $|w| \leqq C_{2}<A$ ) which is a good contour for the phase in (2.11). We omit the proof because it is identical to the proof of the Lemma 2.5 [by (2.32)]. Then by (2.32) and [Sj, p. 23] $P_{\varepsilon}: H_{\psi_{t}, 0} \rightarrow H_{\psi_{t}, 0}$.

Proof of the Theorem. We have:

$$
\begin{aligned}
P_{\varepsilon}\left(z, \partial_{z}, h\right)(E(t) u)(z)= & (2 \pi h)^{-2 n} \int_{\substack{(w, \theta) \in \Gamma_{1} \\
(x, \xi) \in \Gamma_{0}}} e^{i h^{-1}(\langle z-w, \theta\rangle+S(t, w, \xi)-\langle x, \xi\rangle)} \\
& \times a(z, \theta, \varepsilon, h) a_{N_{1}}(t, w, \xi) u(x) d(w, \theta, x, \xi),
\end{aligned}
$$

where $d(w, \theta, x, \xi)=d w \wedge d \theta \wedge d x \wedge d \xi$

$$
=(2 \pi h)^{-n} \int_{\Gamma_{0}} e^{-i h^{-1}\langle w, \xi\rangle} A_{\varepsilon}(z, \xi, t) u(w) d w \wedge d \xi
$$

so that $\left(i h \partial_{t}-P_{\varepsilon}\left(z, \partial_{z}, h\right)\right) E(t) u(z)=F(t, z, h)$, where $\quad$ by $\quad(1.15-17) \quad$ and Proposition 2.4:

$$
|F(t, z, h)| \leqq C e^{h^{-1} \psi_{t}(z)} h^{N_{1}} .
$$

To conclude the proof of the theorem it is enough to apply the stationary phase formula to $E(t) u$ and to estimate the error term. In order to avoid the use of the Morse coordinates, not directly known, we add and subtract in the phase of (2.33) precisely those terms which build a quadratic critical point at $w(t, z)$. The price to pay is of course the inapplicability of the general statement on the smallness of the 
remainder; therefore this property has to be directly proved. Thus we write:

$$
\begin{aligned}
& E(t) u(z)=(2 \pi h)^{-n} \iint_{\Gamma_{0}} e^{-i h^{-1}(\langle w-w(t, z), \xi\rangle-\langle w-w(t, z),-i \bar{w}(t, z)\rangle)} \\
& \quad \times e^{i h^{-1}(S(t, z, \xi)-\langle w,-i \bar{w}(t, z)\rangle-\langle w(t, z), \xi\rangle+\langle w(t, z),-i \bar{w}(t, z)\rangle)} a_{N_{1}}(t, z, \xi, h) u(w) d w \wedge d \xi \\
& =(2 \pi h)^{-n} \int_{|w-w(t, z)| \leqq C_{1}} e^{-h^{-1}|w-w(t, z)|^{2}}\left(e^{i h^{-1}(S(t, z,-i \bar{w})-\langle w(t, z),-i \bar{w}\rangle)} a_{N_{1}}(t, z,-i \bar{w})\right. \\
& \left.\quad \times e^{i h^{-1}(-\langle w,-i \bar{w}(t, z)\rangle+\langle w(t, z),-i \bar{w}(t, z)\rangle)}\right) u(w) L(d w) \\
& =e^{i h^{-1} S(t, z,-i \bar{w}(t, z), \varepsilon)-h^{-1}|w(t, z)|^{2}} u(w(t, z)) \sum_{|\alpha|=0}^{N-1} \frac{1}{\alpha !} \partial_{\xi}^{\alpha} a_{0}(t, z,-i \bar{w}(t, z))(i \bar{w}(t, z))^{\alpha} \\
& \quad+\sum_{l=1}^{N-1} h^{l} \sum_{j=0}^{N_{1}} h^{j} M_{l}\left(a_{j}(t, z,-i \bar{w}(t, z))\right)+R\left(h^{-1}, t, z\right),
\end{aligned}
$$

where

$$
\left|M_{l}\left(a_{j}\right)\right| \leqq \text { const } e^{h^{-1} \psi_{t}(z)}
$$

Since

$$
\operatorname{Im}\langle w,-i \bar{w}(t, z)\rangle \leqq \frac{1}{2}|w|^{2}+\frac{1}{2}|w(t, z)|^{2}-\frac{1}{4}|| w|-| w(t, z) \|^{2},
$$

by the Taylor formula at second order with respect to $\xi$ we have $(v=-\overline{i(w-w(t, z)))}$

$$
\begin{aligned}
& -\operatorname{Im}(S(t, z,-i \bar{w})-\langle w(t, z),-i \bar{w}\rangle-\langle w,-i \bar{w}(t, z)\rangle)-|w(t, z)|^{2}+\frac{1}{2}|w|^{2} \\
= & -\operatorname{Im}\left(S(t, z,-i \bar{w}(t, z))+\left\langle\nabla_{\xi} S(t, z,-i \bar{w}(t, z)),-\overline{i(w-w(t, z))}\right\rangle\right. \\
& \left.+\frac{1}{2}\left\langle Q_{t, z}(w) v, v\right\rangle\right)+\operatorname{Im}\langle w(t, z),-i \bar{w}\rangle+\operatorname{Im}\langle w,-i \bar{w}(t, z)\rangle-|w(t, z)|^{2} \\
& +\frac{1}{2}|w|^{2} \leqq \psi_{t}(z)-\frac{3}{2}|w(t, z)|^{2}+\frac{1}{2}|w|^{2}+C^{\prime}(\varepsilon)|w-w(t, z)|^{2}+\frac{1}{2}|w|^{2} \\
& +\frac{1}{2}|w(t, z)|^{2}-\left.\frac{1}{4}|| w|-| w(t, z)\right|^{2} \leqq \psi_{t}(z)+\frac{3}{4}|w|^{2}+\frac{1}{2}|w||w(t, z)| \\
& +C^{\prime}(\varepsilon)|w-w(t, z)|^{2} \leqq \psi_{t}(z)+\left(\frac{3}{4}(1+\gamma)^{2}+\frac{1}{2} \gamma(1+\gamma)+C^{\prime}(\varepsilon)\right) C_{1}^{2} .
\end{aligned}
$$

Moreover, by our choice of $N$ :

$$
\begin{aligned}
& e^{h^{-1} C_{3}^{2}\left(1-2 \log \left(C_{3}^{2} / C_{1}\right)\right)}\left|R\left(h^{-1}, t, z\right)\right| \\
& \leqq C \max _{w}\left|e^{i h^{-1}(S(t, z,-i \bar{w})-\langle w(t, z),-i \bar{w}\rangle-\langle w,-i \bar{w}(t, z)\rangle+\langle w(t, z),-i \bar{w}(t, z)\rangle)} u(w)\right| .
\end{aligned}
$$

This implies:

$$
\begin{gathered}
\left|R\left(h^{-1}, t, z\right)\right| \leqq C e^{h^{-1} \psi_{t}(z)} e^{-h^{-1} C_{3}^{2}\left(1-2 \log \left(C_{3} / C_{1}\right)\right)} e^{h^{-1}\left(\frac{3}{4}(1+\gamma)^{2}+\frac{1}{2} \gamma(1+\gamma)+C^{\prime}(\varepsilon)\right) C_{1}^{2}} \\
\leqq C e^{h^{-1} \psi_{t}(z)} e^{-\mathrm{const} \cdot h^{-1}}
\end{gathered}
$$

for $\varepsilon$ small enough. This concludes the proof of the theorem.

\section{References}

[A] Arnold, V.I.: Ordinary differential equations. Moscow: MIR Press 1973

[Ba] Bargmann, V.: On a Hilbert space of analytic functions and an associated integral transform. Commun. Pure Appl. Math. 14, 187-214 (1961)

[Ch] Chazarain, J.: Spectre d'un hamiltonien quantique et mécanique classique. Commun. Part. Diff. Eqs. 5, 595-644 (1980) 
[GeSj] Gérard, C., Sjöstrand, J.: Resonances en limite semiclassique et exposants de Lyapunov. Prépublications de l'Université de Paris-Sud, Orsay, 1987

[GrPa] Graffi, S., Paul, T.: The Schrödinger equation and canonical perturbation theory. Commun. Math. Phys. 108, 71-87 (1987)

[GrSj] Grigis, A., Sjöstrand, J.: Front d'onde analytique et sommes de carrés de champs de vecteurs. Duke Math. J. 52, 35-51 (1985)

[HeRo1] Helffer, B., Robert, D.: Comportement semi-classique du spectre des Hamiltonien quantiques elliptiques. Ann. Inst. Fourier 31, 169-223 (1981)

[HeRo2] Helffer, B., Robert, D.: Asymptotique des niveaux d'energie pour des hamiltoniens à un degré de liberté. Duke Math. J. 49, 853-868 (1982)

[LaSj] Lascar, B., Sjöstrand, J.: Equation de Schrödinger et propagation pour de O.D.P. à characteristiques reelles de multeplicité variable. II. Commun. Part. Diff. Eq. 10, 467-523 (1985)

[Le] Lebeau, G.: Deuxième microlocalisation sur les sous-varietés isotropes. Ann. Inst. Fourier (Grenoble) 35, 145-216 (1985)

[N] Narasimhan, R.: Analysis on real and complex manifolds. Amsterdam: North-Holland 1968

[RoPe] Robert, D., Petkov, V.: Asymptotique semiclassique du spectre d'Hamiltoniens quantiques et trajectoires classiques périodiques. Commun. Part. Diff. Eq. 10, 365-390 (1985)

[RoTa] Robert, D., Tamura, H.: Semiclassical bounds for the resolvents of Schrödinger operators and asymptotics of the scattering phase. Commun. Part. Diff. Eq. 9, 1017-1055 (1984)

[Sj] Sjöstrand, J.: Singularités analytiques microlocales. Asterisque 95 (1982)

[Ta] Tamura, H.: Asymptotic formulas with sharp remainder estimates for the bound states of Schrödinger operators. J. Analyse Math. 41, 85-108 (1982)

[Ya1] Yajima, K.: The quasi-classical limit of quantum scattering theory. I. Commun. Math. Phys. 69, 101-129 (1979)

[Ya2] Yajima, K.: The quasi-classical limit of quantum scattering theory. II. Duke Math. J. 48, 1-22 (1981)

[Ze] Zelditch, S.: Reconstruction of singularities for solutions of Schrödinger's equation. Commun. Math. Phys. 90, 1-26 (1983)

Communicated by G. Parisi

Received February 13, 1989 
